\title{
Metodología para la evaluación del nivel tecnológico del cultivo de Rye grass en los Andes ecuatorianos, microcuenca del río Chimborazo
}

\author{
Technological Level Evaluation Methodology of Rye grass cultivation in \\ Ecuadorian Andes, the Chimborazo River watershed
}

Eduardo Antonio Muñoz Jácome

Escuela Superior Politécnica de Chimborazo, México emunoz@espoch.edu.ec

María José Coello

Escuela Superior Politécnica de Chimborazo, México mjose171988@yahoo.com

Fabricio Moreno

Escuela Superior Politécnica de Chimborazo, México fabricio_naki@hotmail.com

César Cruz Escuela Superior Politécnica de Chimborazo, México cero_2d@hotmail.com

\section{Resumen}

La microcuenca del Río Chimborazo está ubicada en la Parroquia San Juan, provincia de Chimborazo, en la zona central de los Andes ecuatorianos. El $90 \%$ de los habitantes son indígenas y hablan el Kichwa. Su extensión es de 12460 hectáreas, de las cuales $27 \%$ son pastizales. En promedio, cada vaca produce 6 litros de leche al día, lo cual se debe entre otros motivos a la mala calidad de los pastos. Se desconoce el motivo de esto último, así que se llevó a cabo un estudio que permite idear una metodología sencilla y participativa que determine el nivel tecnológico del cultivo y establezca científicamente la problemática en la implementación y manejo del pasto. Asimismo, con ello se busca definir si la tecnología agronómica hasta hoy desarrollada y aplicada en dicha área andina es sostenible.

La presente investigación se erige como base orientadora para futuros trabajos científicos agronómicos, de capacitación y asistencia técnica a desarrollar, con un enfoque de sostenibilidad 
para las diferentes prácticas y fases del cultivo. Después del análisis correspondiente se reportan las deficiencias en las fases agronómicas del mismo.

Palabras clave: Niveles tecnológicos, Rye Grass, manejo eficiente de cultivos, Lolium multiflorum, microcuenca, río Chimborazo.

\section{Abstract}

The watershed of the river Chimborazo is located in Parroquia San Juan, province of Chimborazo, in the central zone of the Ecuadorian Andes. $90 \%$ of the inhabitants are indigenous and speak the Kichwa. Its extension is 12,460 hectares, of which $27 \%$ are grasslands. On average, each cow produces 6 liters of milk a day, due partly to the poor quality of the pastures. The reason for this last, is unknown so it was conducted a study allowing to devise a simple and participatory methodology which determine the technological level of the crop and scientifically set the problems in the implementation and management of pasture. Also, this is seeks to define if the agronomic technology so far developed and applied in the Andean area is sustainable.

This research stands as a guiding basis for future agronomic scientific work, training and technical assistance to develop, with a focus on sustainability to different practices and phases of the crop. After the corresponding analysis deficiencies in agronomic phases are reported.

Key words: Technological levels, Rye Grass, efficient management of crops, Lolium multiflorum, watershed, Chimborazo River.

Fecha recepción: Noviembre 2014

Fecha aceptación: Mayo 2015

\section{Introducción}

Según el III Censo Agropecuario Nacional 2000, 25 \% de la superficie total de la tierra está cubierta por pastos. En el Ecuador se reveló que $41 \%$ del suelo de uso agropecuario se destina a pastos; y que entre 1974 y 2000 estas áreas se han incrementado en $70 \%$, es decir, en las últimas décadas ha existido una clara tendencia a la producción ganadera. En la sierra esto se explica por el alto riesgo de la agricultura, mientras que la ganadería de leche representa ingresos estables. En la costa y en la 
Amazonía, la incorporación de pastizales para la ganadería se hace con fines económicos, ocasionando serios problemas relacionados con la tala de bosques.

Según INEC 2010, en el país había 12355000 ha productivas, de las cuales $38 \%$ corresponden a pastos. El uso del suelo que se maneja a nivel nacional para el desarrollo de potreros naturales es de $25.2 \%$ y de potreros cultivados es de $74.8 \%$.

La microcuenca del río Chimborazo se encuentra ubicada en los andes ecuatorianos, está formada por una población que en $93 \%$ se reconoce como indígena y que es Kichwa parlante, perteneciente a la nacionalidad Puruhá.

La producción lechera en la microcuenca del río Chimborazo es de 6 litros por vaca y por día en promedio, esto se debe entre otros aspectos a la mala calidad de los pastos, sin embargo, se desconocen los motivos que generan esta situación, por lo que fue necesario realizar un estudio que permita crear una metodología para determinar el nivel tecnológico del cultivo y establecer científicamente la problemática en la implementación y manejo de este importante pasto para definir si es sostenible o no con la tecnología agronómica hasta hoy desarrollada y aplicada en dicha área geográfica andina.

La presente investigación constituye la base orientadora de los trabajos científicos agronómicos, de capacitación y asistencia técnica a desarrollar en el futuro con un enfoque de sostenibilidad para las diferentes prácticas y fases del cultivo. Esto permitió determinar la sostenibilidad del cultivo del Rye Grass a través de una metodología que evalúa su nivel tecnológico en la microcuenca del río Chimborazo, parroquia San Juan, provincia de Chimborazo, Ecuador. Así, se definió teóricamente el manejo eficiente del cultivo de Rye Grass, determinando el nivel tecnológico de cultivo practicado por los agricultores en el área de estudio, y diseñando una propuesta para el mejoramiento tecnológico del cultivo. 


\section{Método}

\section{Localización}

\section{Ubicación geográfica}

La microcuenca del río Chimborazo está dentro de los siguientes límites geográficos:

Latitud: $9825460 \mathrm{~N}$

Longitud: $746667 \mathrm{E}$

Altitud. 3300 m.s.n.m.

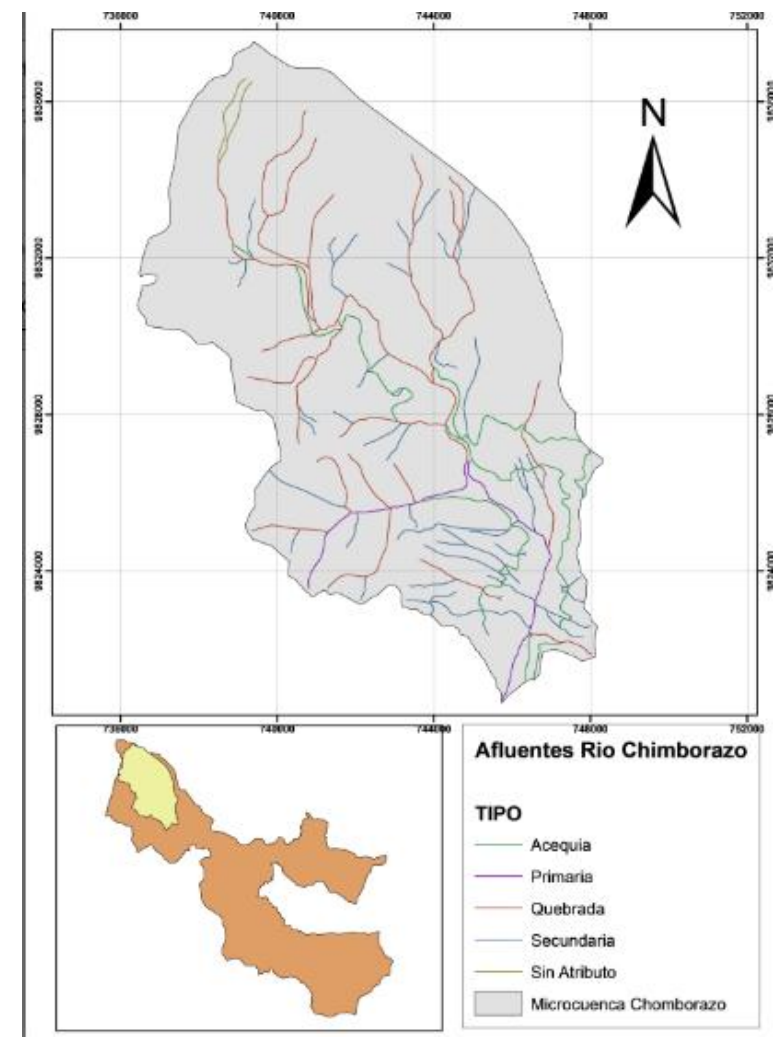

Figura 1. Mapa de hidrografía de la microcuenca del río Chimborazo.

Fuente: MAGAP 2012

Elaborado por: Eduardo Muñoz, César Cruz

\section{Características climáticas}

Según el gobierno autónomo descentralizado de la Parroquia San Juan (2012), las características climáticas son:

Temperatura: $12^{\circ}-16^{\circ} \mathrm{C}$

Precipitación: 500 - 1000mm

Humedad relativa: 80 a $90 \%$ 


\section{Zonas de vida}

El área motivo de la presente investigación pertenece a las zonas de vida:

a. Bosque seco montano bajo (bsMB), esta zona de vida se ubica entre los 2900 y $3200 \mathrm{msnm}$.

b. Bosque húmedo montano bajo (bhMB), esta zona de vida se ubica entre los 3200 y 3600 msnm.

\section{Características del suelo}

\section{Taxonomía del suelo}

Tabla 1. Órdenes de suelo en la microcuenca del río Chimborazo

\begin{tabular}{|c|c|c|}
\hline Orden & Extensión (Ha) & Porcentaje (\%) \\
\hline Entisol & 625 & 5,0 \\
\hline Incetisol & 6200 & 49,6 \\
\hline Mollisol & 8 & 0,1 \\
\hline Histosol & 2210 & 17,7 \\
\hline Nieve & 925 & 7,4 \\
\hline Eriales & 2522 & 20,2 \\
\hline Total & 12490 & 100,0 \\
\hline
\end{tabular}

Fuente: MAGAP 2012

Elaborado por: Eduardo Muñoz, César Cruz 


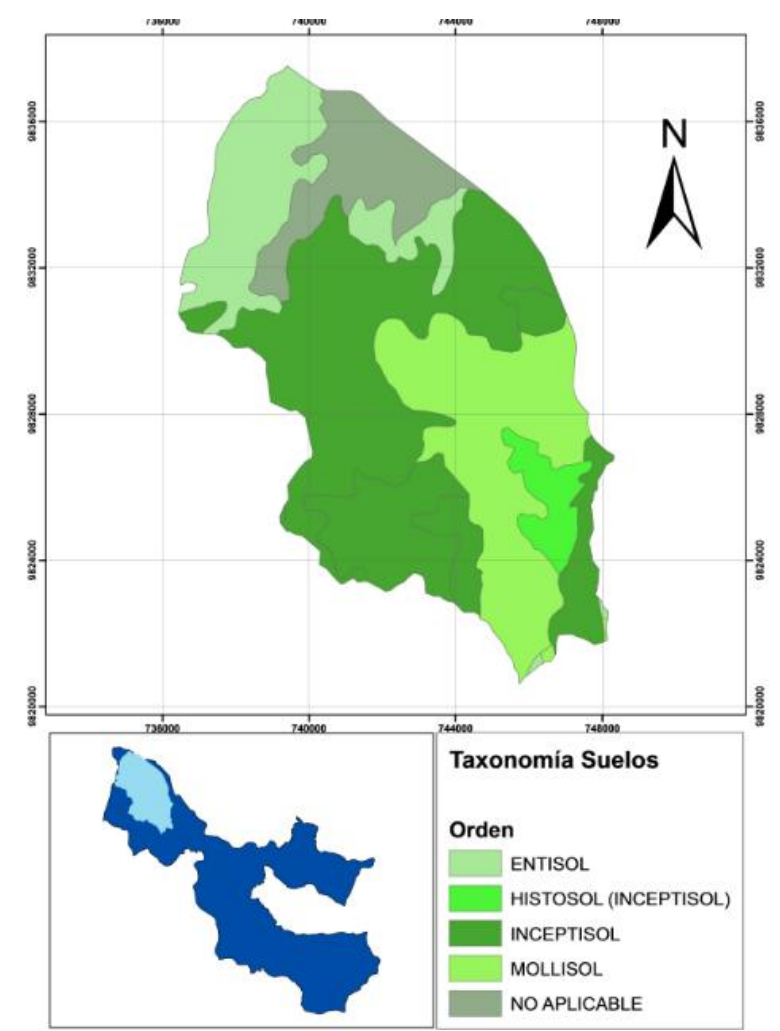

Figura 2. Mapa de Taxonomía de suelos de la microcuenca del río Chimborazo Fuente: MAGAP 2012

Elaborado por: Eduardo Muñoz, César Cruz 


\section{Uso actual del suelo}

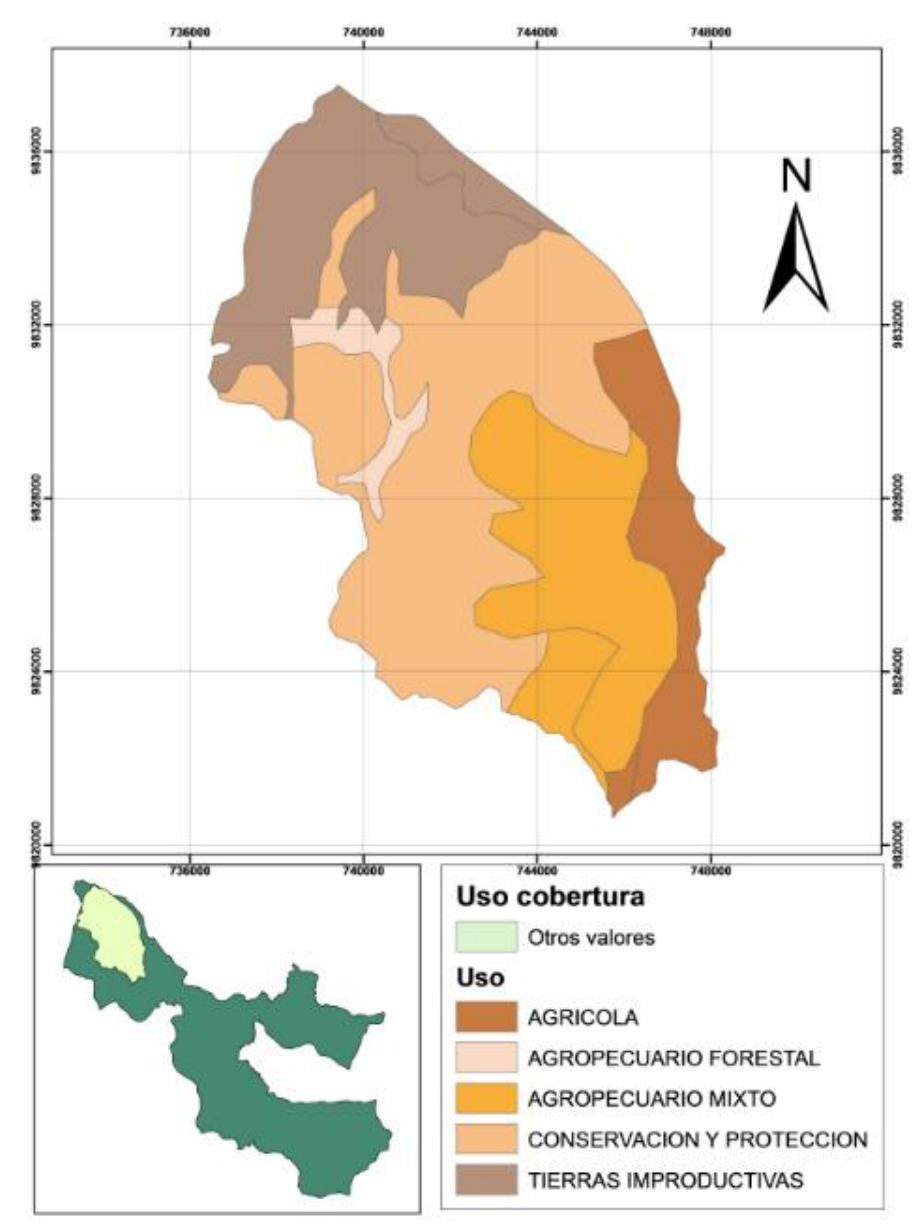

Figura 3. Mapa de uso actual del suelo de la microcuenca del río Chimborazo.

Fuente: MAGAP 2012

Elaborado por: Eduardo Muñoz y César Cruz

\section{Método para la definición teórica para el manejo eficiente del cultivo de Rye grass}

Se aplicaron los métodos teóricos basados en la recolección de información sobre el manejo eficiente del cultivo de Rye Grass, considerando las prácticas agronómicas para las fases siembra y manejo del pastoreo de este cultivo.

Método para determinación del nivel tecnológico del cultivo de Rye grass practicado por los agricultores en el área de estudio

A partir de la base teórica se diseñó una metodología que permitió el análisis del nivel tecnológico del cultivo de Rye Grass, considerando como variables las actividades y las fases de producción. En concreto, a partir del sustento teórico científico se elaboró la tabla óptima o de eficiencia que permite confrontar el manejo ideal del cultivo frente a la práctica real de los agricultores, determinando los problemas en cada una de sus partes. 
Se emplearon los métodos empíricos de la investigación científica con la aplicación de técnicas estadísticas que permitieron el análisis por práctica o actividad agrícola asociada a cada fase, por fases y del cultivo en general.

Se trabajó considerando como universo los productores del cultivo de Rye Grass pertenecientes a la parte baja y media de la Microcuenca del Rio Chimborazo. Para lograr la representatividad de la muestra, se la estratificó.

\section{Método para el diseño de una propuesta que mejore la tecnología del cultivo}

En forma participativa con los agricultores se hicieron: el análisis de problemas, de objetivos, se definieron alternativas y se diseñó la matriz del Marco Lógico.

\section{Resultados y discusión}

Manejo eficiente del cultivo de Rye grass desde el análisis teórico.

\section{Clasificación taxonómica}

$\begin{array}{ll}\text { Reino: } & \text { Plantae } \\ \text { División: } & \text { Magnoliophyta } \\ \text { Clase: } & \text { Liliopsida } \\ \text { Orden: } & \text { Cyperaceae }\end{array}$

Familia: Poaceae

Subfamilia: Pooideae

Tribu: $\quad$ Poeae

Género: Lolium

Especie: $\quad$ L. multiflorum (Alarcón, A. 2007).

\section{Descripción botánica}

\section{Hábito y forma de vida}

Planta herbácea anual, bianual o perenne (Rocalba, 2011). 


\section{Tamaño}

De hasta 1 (1.3) m de alto (Rocalba, 2011).

\section{Tallo}

Cespitoso (forma matas aglomeradas), erecto o doblado en los nudos (Rocalba, 2011).

\section{Hojas}

Vainas foliares con aurículas (orejas) conspicuas hacia el ápice; lígulas de 1-4 mm de largo; lámina de hasta $22 \mathrm{~cm}$ de largo y $8 \mathrm{~mm}$ de ancho, lisas en el envés, opacas y ásperas en el haz (Rocalba, 2011).

\section{Inflorescencias o espiguillas}

Solitarias, sésiles, alternas, de 10 a $20 \mathrm{~mm}$ de largo con 4 a 22 flores; glumas de 5 a $10 \mathrm{~mm}$ de largo, 5 a 7 nervadas, la inferior ausente, la segunda opuesta al raquis y más corta que la mitad de la longitud de la espiguilla; lema de 4 a $8 \mathrm{~mm}$ de largo, 5-7 nervada, redondeada en el dorso, de bordes algo ásperos, con arista subapical de 0 a $15 \mathrm{~mm}$ de largo; semilla de $\pm 4 \mathrm{~mm}$ de largo (Rocalba, 2011).

\section{Zonificación del Rye Grass}

El Rye Grass es considerado la mejor opción forrajera en las zonas de clima templado por sus altos rendimientos, calidad nutritiva y habilidad para crecer en gran diversidad de suelos (Alarcón, A. 2007).

El Rye Grass tiene un alto rango de adaptación a los suelos, prefiriendo los fértiles con buen drenaje. Tolera periodos largos de humedad, así como suelos ácidos y alcalinos (pH 5.5 a 7.8); cuando este es menor que 5.0, la toxicidad por aluminio puede ser un problema. Realiza un aporte nutricional de 12 a $18 \%$ de proteína cruda en la alimentación del ganado (Alarcón, A. 2007). 


\section{Variedades}

\section{Rye Grass Inglés}

El Rye Grass Inglés es una excelente gramínea forrajera que se desarrolla perfectamente en tierras frescas y sanas. No tolera la sequía ni las altas temperaturas. Soporta muy bien el pisoteo y su forraje es muy apetecido por todo tipo de ganado. Con un manejo adecuado su presencia en la pradera puede ser superior a los cuatro años, hasta los seis años. Presenta una altura de planta al inicio de la floración de 30 a 35 cm (Rocalba, 2011).

\section{Rye Grass Italiano}

El Rye Grass Italiano es la gramínea forrajera más utilizada de todas. Es una especie bianual que puede durar hasta 5 años con un manejo adecuado. Es parecida al Rye Grass Inglés pero con hojas más largas y anchas, con un color verde más claro. La espiga tiene aristas. Tiene una fácil implantación y se comporta de forma muy agresiva. Necesita suelos fértiles para mostrar todo su potencial productivo. La altura de planta al inicio de la floración es de 50 a 60 cm (Rocalba, 2011).

\section{Rye Grass Westerwold}

Se utiliza siempre en siembras puras para el establecimiento de praderas de corta duración y máxima producción en el menor tiempo. Muy utilizado en regadíos para producir gran cantidad de forraje y dejar libre pronto el terreno para poder establecer otro cultivo. Produce un forraje de Alta Calidad que puede utilizarse tanto para pastoreo como ensilado, así como para henificado. Presenta una altura de planta al inicio de la floración de 50 a 60 cm (Rocalba, 2011).

\section{Rye Grass Híbrido}

Es el resultado del cruzamiento entre un Rye Grass Inglés y un Rye Grass Italiano, por lo que esta especie presenta caracteres intermedios de ambas. Del Rye Grass Italiano recibe su envergadura y alta productividad y del Rye Grass Inglés la perennidad, la cual es de 3 años. La altura de planta al inicio de la floración es de 50 a 60 cm (Rocalba, 2011). 


\section{Requerimientos edafoclimáticos}

El Rye Grass tiene un alto rango de adaptación a los suelos, prefiriendo los fértiles con buen drenaje. Tolera períodos largos de humedad (15 a 20 días), así como suelos ácidos y alcalinos (pH 5.5 a 7.8); cuando este es menor que 5.0, la toxicidad por aluminio puede ser un problema (Alarcón, A. 2007).

Clementeviven, (2010) indica que el Rye Grass se adapta muy bien a los climas fríos, con veranos de días cálidos y noches frescas, crece en todo tipo de suelos, tolerando hasta los suelos pesados, pero en terrenos húmedos y fértiles es donde mejor vegeta, siendo una especie altamente exigente en agua y Nitrógeno, que no se adapta bien a la sequía y es muy poco tolerante a la sombra.

La Universidad Pública de Navarra (2011), reporta que el Rye Grass se adapta bien en climas templado-húmedos. Tolera el frío moderado pero es sensible al calor y a la sequía. Su crecimiento se ralentiza a partir de $\operatorname{los} 25^{\circ} \mathrm{C}$ y se paraliza a los $35^{\circ} \mathrm{C}$. Se adapta a un amplio rango de suelos. Presenta una buena respuesta a la fertilización nitrogenada, en terrenos ricos en nitrógeno se desarrolla profusamente, pudiendo dominar el pasto. Soporta la compactación pero no tolera el encharcamiento.

El SIAN (2011), señala que el Rye Grass es cultivado en altitudes comprendidas entre 2200 y 3000 m.s.n.m., aun cuando en investigaciones realizadas por el Instituto Nacional de Investigaciones Agropecuarias de Venezuela, ha demostrado gran desarrollo y vigor en alturas entre 3100 y 3500 metros.

\section{Implantación}

Clementeviven, (2010) señala que el Rye Grass presenta una implantación rápida, germina entre los 5 y 7 días después de la siembra, pasando de inmediato a establecerse y a proteger el suelo.

La Universidad Pública de Navarra (2011), señala que su crecimiento inicial no es tan rápido como el del Rye Grass italiano pero sí superior al resto de gramíneas pratenses de la zona templada; presentando además una persistencia de 4-5 años o más si las condiciones de medio son favorables. 


\section{Densidad de siembra}

La Unión Ganadera Regional de Jalisco, (2011), reporta que para asegurar el establecimiento de la pradera se recomienda utilizar semilla certificada de Rye Grass, lo que en semilla comercial significa sembrar de 30-10 kg de semilla por hectárea.

Ducos, E. (2011), indica que la siembra se realiza a una dosis de $20-24 \mathrm{~kg} / \mathrm{ha}$. Cuando se siembra puro o asociado con trébol blanco, y en dosis proporcionalmente menores según la relación que se quiera establecer con otra gramínea.

Sian (2011), señala que el Rye Grass se siembra al voleo utilizando de 25 a $30 \mathrm{~kg} / \mathrm{ha}$ de semilla cuando se emplean Rye Grass naturales, o de 30 a $35 \mathrm{~kg} / \mathrm{ha}$ de semilla cuando se siembran Rye Grass híbridos.

\section{Métodos de siembra}

La forma o método de siembra dependerá de la maquinaria y equipo de que se disponga, aunque puede ser manual, al voleo o con una sembradora ciclónica manual. También puede utilizarse una voleadora de fertilizante calibrada adecuadamente, efectuando el tapado de la semilla mediante un paso de rastra de ramas procurando que la semilla quede a una profundidad de $1.0-1.5 \mathrm{~cm}$. de la superficie del suelo (Unión Ganadera Regional de Jalisco, 2011).

\section{Riego}

Durante el periodo de establecimiento, el primer riego se aplicará después de la siembra; deberá ser pesado y cuando sea por gravedad, cuidar que este no arrastre la semilla. El segundo riego se realizará a los 8-11 días, el tercero a los 10-15 días. y el cuarto riego de los 15-20 días. Esta frecuencia de riegos dependerá de la textura del suelo; en los suelos arenosos deberá ser más frecuente y en el caso de suelos arcillosos, los cuales tienen mayor capacidad de retención de humedad, se deberá cuidar que el terreno no se encostre, principalmente durante los tres primeros riegos (Unión Ganadera Regional de Jalisco, 2011). 


\section{Fertilización}

La Unión Ganadera Regional de Jalisco, (2011), indica que para la fertilización a la siembra se recomienda aplicar $80 \mathrm{~kg}$ de nitrógeno y $60 \mathrm{~kg}$ de fósforo por hectárea. Lo que equivale a $175 \mathrm{~kg}$ de urea y $130 \mathrm{~kg}$ de superfosfato triple por hectárea. La aplicación del fertilizante se realiza al voleo y se incorpora al suelo con el agua de riego. Durante el período de utilización de la pradera, se realizará después de cada corte o pastoreo (aproximadamente cada 25-30 días); aplicando al voleo o con el agua de riego, $50 \mathrm{~kg}$ de nitrógeno por hectárea, lo que equivale a aplicar 100-110 kg de urea por hectárea.

El Sian (2011), recomienda aplicar 350 kg de nitrógeno más 50 a 100 kg/ ha de fósforo y potasio por año. Con un buen programa de fertilización se logran producciones de 18 a 20 tn de materia verde por hectárea por año, equivalente a 9 o10 tn de forraje seco por año.

\section{Rotación de potreros}

El concepto de rotación se basa en que la pradera, luego de ser pastoreada, utiliza los nutrientes de reserva para recuperarse y tiene necesidad de descansar lo suficiente para volver a almacenarlos, porque de lo contrario se agota (Valencia, E. 2010).

De lo anterior se desprende que el periodo de rotación tiene dos partes, el periodo de pastoreo (o de ocupación) y el de descanso (Valencia, E. 2010).

Adicionalmente hay 4 leyes que se aplican al pastoreo en rotación:

- $\quad$ El periodo de descanso debe ser lo suficientemente largo.

- $\quad$ El periodo de ocupación debe ser corto.

- $\quad$ El pasto de mejor calidad lo deben cosechar los animales con mayores requerimientos.

- Una vaca lechera no debe permanecer más de 3 días en cada potrero; el ideal es 1 día (Valencia, E. 2010). 


\section{Utilización de la pastura}

El forraje siempre debe ser pastoreado y consumido en su punto óptimo: para el caso del Rye Grass se considera 20 a $25 \mathrm{~cm}$ de altura. Etapa en la que hay más cantidad de hojas y la caña no es dura (Masal, 2009).

No debe pastorearse el pasto por debajo de 5 a $7 \mathrm{~cm}$. de altura, a fin de favorecer un rebrote más rápido (Masal, 2009).

\section{Técnicas de pastoreo}

\section{1) Pastoreo continuo}

Cuando se utiliza el pastoreo continuo, es decir los animales disponen de un solo potrero en el cual pastorean durante todo el año, es común que se presenten problemas por sobre pastoreo o sub pastoreo (Masal, 2009).

El sobre pastoreo ocurre cuando se pastorea un sitio demasiado tiempo con un número grande de animales. Los animales comen todo lo que pueden disminuyendo las reservas de los pastos, lo que no permite su rápida recuperación (Masal, 2009).

El sub pastoreo ocurre cuando hay pocos animales en el potrero, estos consumen las partes más tiernas y los brotes del pasto, dejando los tallos. Como consecuencia, los pastos "envejecen", su calidad nutritiva disminuye y los animales no los consumen (Masal, 2009).

\section{2) Pastoreo por estaca}

Esta forma de pastoreo es la que mejor se acomoda a los pequeños criadores de ganado vacuno en las comunidades que tienen chacras en laderas o tienen muy poco pasto instalado (Masal, 2009).

Las vacas se amarran con una soga de unos 6 metros de largo, avanzando la estaca tres veces, unos tres metros cada vez. Este sistema de pastoreo puede ser utilizado cuando se dispone de menos de 5 vacas (Masal, 2009). 


\section{3) Pastoreo por franjas}

Esta recomendación es para los ganaderos que tienen pastos instalados en gran cantidad y en zonas planas o cuando se cuenta con más de cinco vacas; el sistema que utiliza cercos eléctricos es el más conveniente (Masal, 2009). Cada día se mueve dos o más veces la cerca eléctrica para dar cada vez una franja nueva de pasto a los animales, hasta haber pastoreado todo el sitio y pasar a uno nuevo.

Esto implica:

- Trabajo adicional para el manejo de la cerca eléctrica.

- $\quad$ Un equipo e instalación correcta (Masal, 2009).

\section{4) Pastoreo rotativo}

Esta recomendación es para empresas campesinas y ganaderos que están en condiciones de invertir en mallas además de contar con gran cantidad de animales. Se divide toda la pastura en lotes o potreros, en los cuales los animales permanecen 1 o 2 días como máximo, pasando al siguiente. La ventaja de este sistema es que se pueden llevar a cabo prácticas de abono, riego, corte para emparejar el pasto, y otras sin molestar a los animales, obteniéndose pasto más tierno y de mejor calidad (Masal, 2009).

\section{Nivel tecnológico del cultivo de rye grass en la microcuenca del rio Chimborazo}

Para determinar el nivel tecnológico del cultivo en la microcuenca se identificó: 


\section{Universo}

Tabla 1. Número de familias por comunidad que cultivan Rye Grass.

\begin{tabular}{|c|c|}
\hline \multicolumn{2}{|c|}{ PARTE BAJA } \\
\hline Organización Comunitaria & Número de familias \\
\hline Comunidad Pisicaz Alto y Bajo & 60 \\
\hline Asoc. Cantarilla & 28 \\
\hline \multicolumn{2}{|c|}{ PARTE MEDIA } \\
\hline Organización Comunitaria & Número de familias \\
\hline Comunidad Chaupi Pomalo & 43 \\
\hline Comunidad Calera Grande & 90 \\
\hline Comunidad Calera Yumi & 28 \\
\hline Comunidad Calerita Santa Rosa & 42 \\
\hline Comunidad Shobol Llinllin & 78 \\
\hline Comunidad Calera Shobol Pamba & 72 \\
\hline Comunidad Guabug & 132 \\
\hline TOTAL & 572 \\
\hline
\end{tabular}

Fuente: Plan de Desarrollo y Ordenamiento Territorial - Parroquia San Juan, 2012. Elaborado Por: Muñoz, E, Moreno, F y Coello, MJ, Cruz César 2013.

\section{Muestra}

Para el cálculo de la muestra se utilizó la siguiente fórmula:

$$
\begin{gathered}
n=\frac{N \times p \times q}{(N-1) \times\left(\frac{e}{z}\right)^{2}+p \times q} \\
n=\frac{572 \times 0,5 \times 0,5}{(571) \times\left(\frac{0,05}{1,96}\right)^{2}+0,5 \times 0,5} \\
n=229
\end{gathered}
$$

\section{Estratificación}

Para el cálculo de la estratificación se utilizó la siguiente fórmula:

$$
(f)=\frac{n}{N}=\frac{229}{572}=0,40
$$


Tabla 2. Estratificación de la muestra por comunidades que cultivan Rye Grass.

\begin{tabular}{|l|l|l|}
\hline \multicolumn{3}{|c|}{ PARTE BAJA } \\
\hline Organización Comunitaria & Número de familias & Estratificación \\
\hline Comunidad Pisicaz Alto y Bajo & 60 & 24 \\
\hline Asoc. Cantarilla & 28 & 11 \\
\hline PARTE MEDIA & \\
\hline Organización Comunitaria & Número de familias & Estratificación \\
\hline Comunidad Chaupi Pomalo & 43 & 17 \\
\hline Comunidad Calera Grande & 90 & 36 \\
\hline Comunidad Calera Yumi & 28 & 11 \\
\hline Comunidad Calerita Santa Rosa & 42 & 17 \\
\hline Comunidad Shobol Llinllin & 78 & 31 \\
\hline Comunidad Calera Shobol Pamba & 72 & 29 \\
\hline Comunidad Guabug & 132 & 53 \\
\hline TOTAL & $\mathbf{5 7 2}$ & $\mathbf{2 2 9}$ \\
\hline
\end{tabular}

Elaborado Por: Muñoz, E, Moreno, F y Coello, MJ, Cruz César 2013.

\section{Variables para determinar el estado del cultivo practicado por los agricultores}

Fundamentándose en la revisión de literatura y considerando el nivel de instrucción de los agricultores, que en el área de estudio en $7 \%$ son analfabetos, en $60 \%$ poseen solo instrucción primaria, y además hablan el idioma Kichwa, las tablas se confeccionaron de tal manera que sean de fácil entendimiento y aplicación

Tabla óptima para la fase siembra

Tabla 3. Variables para la calificación de la fase siembra.

\begin{tabular}{|c|c|}
\hline \multicolumn{2}{|c|}{ Variables para la calificación según práctica o actividad } \\
\hline \multicolumn{2}{|c|}{ Siembra } \\
\hline Variedad Perenne y anual & 1 \\
\hline Densidad de siembra(66- $77 \mathrm{lbs} / \mathrm{ha})$ & 1 \\
\hline Profundidad de siembra $(1-1,5 \mathrm{~cm})$ & 1 \\
\hline Con riego & 1 \\
\hline Fertilización N y P & 1 \\
\hline TOTAL & $\mathbf{5}$ \\
\hline
\end{tabular}

Elaborado Por: Muñoz, E, Moreno, F y Coello, MJ. 2013.

\section{Fundamentos teóricos adoptados para la calificación de la fase siembra}

De acuerdo con Hidalgo, E. (2010)., el Ecuador tiene una gran demanda de semillas de pastos y al no tener producción hace que se deban importar. En el sector agropecuario las principales 
importaciones provienen de países como: Estados Unidos, del cual se ha importado $80.77 \%$ del total de semillas que ingresaron al Ecuador; de Nueva Zelanda se importó $6.27 \%$; y $5.17 \%$ de Holanda.

Rocalba, (2011) indica que se recomienda una mezcla de la variedad perenne y anual.

Sian, (2011) señala que la densidad de siembra va entre $30-35 \mathrm{Kg} / \mathrm{ha}$.

Según Unión Ganadera Regional de Jalisco, (2011) la semilla debe quedar a una profundidad de 1 $1.05 \mathrm{~cm}$ de la superficie del suelo.

Según la Unión Ganadera Regional de Jalisco, (2011) se aplicará un riego después de la siembra.

Con respecto a la fertilización, la Unión Ganadera Regional de Jalisco, (2011) recomienda aplicar nitrógeno y fósforo.

\section{Tabla óptima para la fase pastoreo}

Tabla 4. Variables para la calificación de la fase pastoreo.

\begin{tabular}{|c|c|}
\hline \multicolumn{2}{|c|}{ Criterios de calificación según practica o actividad } \\
\hline \multicolumn{2}{|c|}{ Pastoreo } \\
\hline Duración del pasto (5 - 6 años) & 1 \\
\hline Tiempo de pastoreo (3 días) & 1 \\
\hline Técnicas de pastoreo (estaca/franja/rotativo) & 1 \\
\hline Altura del pasto $(20-25 \mathrm{~cm})$ & 1 \\
\hline Riego en época seca (4 riegos) y fertilización & 1 \\
\hline TOTAL & $\mathbf{5}$ \\
\hline
\end{tabular}

Elaborado por: Muñoz E., Moreno F. y Coello MJ, Cruz César.

\section{Fundamentos teóricos adoptados para la calificación de la fase pastoreo}

Según Rocalba (2011), los Rye Grass Inglés e Italiano en promedio pueden llegar a durar de 5 a 6 años.

Para Valencia, E. (2010), el tiempo de pastoreo no debe ser más de 3 días. 
De acuerdo con Masal (2009), las mejores técnicas de pastoreo son por estaca, por franjas y rotativos, ya que de esta manera no existe el sobrepastoreo.

En cuanto al consumo del forraje, Masal (2009) señala que debe ser consumido cuando tenga una altura de $20-25 \mathrm{~cm}$.

La Unión Ganadera Regional de Jalisco (2011) orienta que en época seca se deben realizar cuatro riegos.

\section{Escalas de calificación}

$5=$ excelente, $4=$ muy bueno, $3=$ bueno; 2 = regular y $1=$ malo

De 1 a 3 la práctica se considera problema; 4 es susceptible de ser mejorado.

Inicialmente y con base en los fundamentos teóricos se observa y califica la aplicación o no de cada una de las actividades que forman parte de cada fase.

\section{Evaluación del nivel tecnológico por fases}

Para determinar el nivel tecnológico por fases y tomando en cuenta los resultados de cada práctica o actividad se usó la escala 5 para excelente, 4 para muy buena, 3 para buena, 2 para regular, 1 para mala, estableciéndose que hasta un puntaje de 3 se considera problema, y 4 es susceptible de ser mejorado.

\section{Evaluación del nivel tecnológico del cultivo en general}

Para evaluar el nivel tecnológico del cultivo en general, se sumó el resultado de las 2 fases estudiadas y se empleó la siguiente escala: 10 excelente, de 8 a 9 muy bueno, de 6 a 7 bueno, de 4 a 5 regular y menor de 4 malo. 


\section{Nivel tecnológico del cultivo}

Tabla 5. Uso de variedades perennes y anuales

\begin{tabular}{|l|l|l|l|l|}
\hline PRÁCTICA & FA & FAA & FR & FRA \\
\hline No usa variedad perenne y anual & 148 & 148 & $65 \%$ & $65 \%$ \\
\hline Sí usa variedad Perenne y anual & 81 & 229 & $35 \%$ & $100 \%$ \\
\hline
\end{tabular}

Elaborado por: Muñoz E., Moreno F. y Coello M.

El $65 \%$ de los agricultores no utiliza variedades de Rye Grass perenne y anual por desconocimiento, mientras que $35 \%$ sí lo hace.

Tabla 6. Aplicación de la densidad adecuada de siembra.

\begin{tabular}{|l|c|c|c|c|}
\hline \multicolumn{1}{|c|}{ PRÁCTICA } & FA & FAA & FR & FRA \\
\hline No aplica la densidad de siembra $(66-77 \mathrm{lb} / \mathrm{a})$ & 221 & 221 & $97 \%$ & $97 \%$ \\
\hline Sí aplica la densidad de siembra $(66-77 \mathrm{lb} / \mathrm{ha})$ & 8 & 229 & $3 \%$ & $100 \%$ \\
\hline
\end{tabular}

Elaborado por: Muñoz E., Moreno F. y Coello M.

El $97 \%$ de los agricultores, que son la mayoría, no toman en consideración la densidad de siembra óptima (67 - 77 lb/ha de semilla), mientras que $3 \%$ sí lo hace.

Tabla 7. Profundidad de siembra recomendada

\begin{tabular}{|l|c|c|c|c|}
\hline \multicolumn{1}{|c|}{ PRÁCTICA } & FA & FAA & FR & FRA \\
\hline $\begin{array}{l}\text { Sí siembra a una profundidad de } \\
1.5 \mathrm{~cm}\end{array}$ & 229 & 229 & $100 \%$ & $100 \%$ \\
\hline
\end{tabular}

Elaborado por: Muñoz E., Moreno F. y Coello M.

El $100 \%$ de los agricultores profundizan adecuadamente la semilla al momento de la siembra (1 $1.5 \mathrm{~cm})$.

Tabla 8. Práctica del riego

\begin{tabular}{|r|c|c|c|c|}
\hline PRÁCTICA & FA & FAA & FR & FRA \\
\hline $\begin{array}{c}\text { No siembra bajo } \\
\text { condiciones de riego }\end{array}$ & 69 & 69 & $30 \%$ & $30 \%$ \\
\hline $\begin{array}{c}\text { Siembra bajo condición } \\
\text { de riego }\end{array}$ & 160 & 229 & $70 \%$ & $100 \%$ \\
\hline
\end{tabular}

Elaborado por: Muñoz E., Moreno F. y Coello M.

El $30 \%$ de los agricultores no realizan el riego después de la siembra, mientras que 70 \% sí lo hace. 


\begin{tabular}{|l|l|l|l|l|}
\hline PRÁCTICA & FA & FAA & FR & FRA \\
\hline No emplea fertilización con N y P & 206 & 206 & $90 \%$ & $90 \%$ \\
\hline Sí emplea fertilización con N y P & 23 & 229 & $10 \%$ & $100 \%$ \\
\hline
\end{tabular}

Tabla 9. Aplicación de fertilización con base en $\mathrm{N}$ y $\mathrm{P}$ en la siembra

$$
\text { Elaborado por: Muñoz E., Moreno F. y Coello M. }
$$

El $90 \%$ de los agricultores no fertilizan el terreno al momento de la siembra, el $10 \%$ sí lo hace.

Tabla 10. Duración del pasto

\begin{tabular}{|c|c|c|c|c|}
\hline PRÁCTICA & FA & FAA & FR & FRA \\
\hline No dura el pasto de 5 a 6 años & 52 & 52 & $23 \%$ & $23 \%$ \\
\hline Sí dura el pasto de 5 - 6 años & 177 & 229 & $77 \%$ & $100 \%$ \\
\hline
\end{tabular}

Elaborado por: Muñoz E., Moreno F. y Coello M.

$\mathrm{Al} 23 \%$ de los agricultores no les dura el pasto de 5 a 6 años, $77 \%$ logra una duración en ese tiempo.

Tabla 11. Tiempo óptimo de pastoreo

\begin{tabular}{|l|l|l|l|l|}
\hline \multicolumn{1}{|c|}{ PRÁCTICA } & FA & FAA & FR & FRA \\
\hline No emplea el tiempo de pastoreo 3 días & 206 & 206 & $90 \%$ & $90 \%$ \\
\hline Sí emplea el tiempo de pastoreo de 3 días & 23 & 229 & $10 \%$ & $100 \%$ \\
\hline
\end{tabular}

$$
\text { Elaborado por: Muñoz E., Moreno F. y Coello M. }
$$

El $90 \%$ de los agricultores pastorea sus animales por 3 días, mientras que $10 \%$ no lo hace.

Tabla 12. Aplicación de las técnicas de pastoreo adecuadas

\begin{tabular}{|l|l|l|l|l|}
\hline PRÁCTICA & FA & FAA & FR & FRA \\
\hline $\begin{array}{l}\text { Sí aplica técnicas de pastoreo } \\
\text { (estaca/franja/rotativo) }\end{array}$ & 229 & 229 & $100 \%$ & $100 \%$ \\
\hline
\end{tabular}

Elaborado por: Muñoz E., Moreno F. y Coello M.

El $100 \%$ de los agricultores sí realiza técnicas de pastoreo (estaca/franja/rotativo), en general usan el método de estaca.

Tabla 13. Manejo de la altura apropiadas del pasto

\begin{tabular}{|l|l|l|l|l|}
\hline \multicolumn{1}{|c|}{ PRÁCTICA } & FA & FAA & FR & FRA \\
\hline No maneja la altura apropiada del pasto $(20-25 \mathrm{~cm})$ & 170 & 170 & $74 \%$ & $74 \%$ \\
\hline Sí maneja la altura apropiada del pasto $(20-25 \mathrm{~cm})$ & 59 & 229 & $26 \%$ & $100 \%$ \\
\hline
\end{tabular}

Elaborado por: Muñoz E., Moreno F. y Coello M.

E1 $74 \%$ de los agricultores realiza una sobre explotación al momento del pastoreo (altura 20 - 25 $\mathrm{cm}$ ), mientras que $26 \%$ realiza un pastoreo adecuado. 
Tabla 14. Riego complementario

\begin{tabular}{|c|c|c|r|r|}
\hline PRÁCTICA & FA & FAA & FR & FRA \\
\hline No riega en época seca (4 riegos) & 128 & 128 & $56 \%$ & $56 \%$ \\
\hline Sí riega en época seca (4 riegos) & 101 & 229 & $44 \%$ & $100 \%$ \\
\hline
\end{tabular}

Elaborado por: Muñoz E., Moreno F. y Coello M.

El $56 \%$ de los agricultores no riega en las épocas secas (4 riegos), mientras que $44 \%$ sí lo hace.

Tabla 15. Análisis del nivel tecnológico de la fase de siembra

\begin{tabular}{|l|l|l|l|l|l|}
\hline \multirow{2}{*}{ Siembra } & EXCELENTE & MUY BUENO & BUENO & REGULAR & MALO \\
\cline { 2 - 6 } & $3 \%$ & $7 \%$ & $25 \%$ & $34 \%$ & $30 \%$ \\
\hline
\end{tabular}

Elaborado por: Muñoz E., Moreno F. y Coello M.

De acuerdo a los criterios, a los resultados de las prácticas y a la escala aplicada, con respecto a las labores en la fase de siembra, $3 \%$ de los agricultores lo realiza de una manera excelente, $7 \%$ muy buena, $25 \%$ buena, $35 \%$ regular y $30 \%$ realiza una mala labor.

Tabla 16. Análisis del nivel tecnológico de la fase manejo o pastoreo

\begin{tabular}{|c|c|c|c|c|c|}
\hline \multirow{2}{*}{ Manejo } & EXCELENTE & MUY BUENO & BUENO & REGULAR & MALO \\
\cline { 2 - 6 } & $10 \%$ & $16 \%$ & $18 \%$ & $33 \%$ & $23 \%$ \\
\hline
\end{tabular}

Elaborado por: Muñoz E., Moreno F. y Coello M.

En cuanto a las labores de la fase de manejo, $10 \%$ de los agricultores lo realiza de una manera excelente, $16 \%$ muy buena, $18 \%$ buena, $33 \%$ regular y $23 \%$ realiza una mala labor.

Tabla 17. Análisis general del nivel tecnológico del cultivo de Rye Grass

\begin{tabular}{|l|l|l|l|l|l|}
\hline CALIFICACIÓN & EXCELENTE & MUY BUENO & BUENO & REGULAR & MALO \\
\hline RANGO & 10 & 8 a 9 & 6 a 7 & 4 a 5 & $<4$ \\
\hline CULTIVO & $3 \%$ & $7 \%$ & $18 \%$ & $33 \%$ & 23 \\
\hline
\end{tabular}

Elaborado por: Muñoz E., Moreno F. y Coello M.

El cultivo de Rye Grass en general y en términos agronómicos reporta los siguientes niveles: $3 \%$ de los agricultores lo realiza de una manera excelente, $7 \%$ muy buena, $18 \%$ buena, $33 \%$ regular y $23 \%$ realiza un mal procedimiento en la siembra y el manejo. Con estos resultados la práctica del cultivo para la mayoría de agricultores es REGULAR, lo que significa que las prácticas resultantes del paquete tecnológico creado desde la revolución verde no han sido adecuadas a la realidad de los agricultores indígenas de la microcuenca del río Chimborazo. 


\section{Propuesta para mejorar el manejo del cultivo de rye grass}

Aplicada la metodología diseñada y con base en los resultados de la evaluación del nivel tecnológico del cultivo, en forma participativa se procedió a trabajar la propuesta, misma que se presenta a continuación.

\section{Análisis de problemas}

Con la participación de los involucrados se determinó como problema central el inadecuado manejo del cultivo, debido a 2 causas principales: malas prácticas en la siembra e inadecuadas prácticas en el manejo; la primera tiene como subcausa la inadecuada profundidad de siembra, malas prácticas de riego e inadecuada fertilización. Las inadecuadas prácticas en el manejo están dadas por uso de pasto viejo, sobrepastoreo y malas prácticas de riego, como se puede observar en la figura 1. 


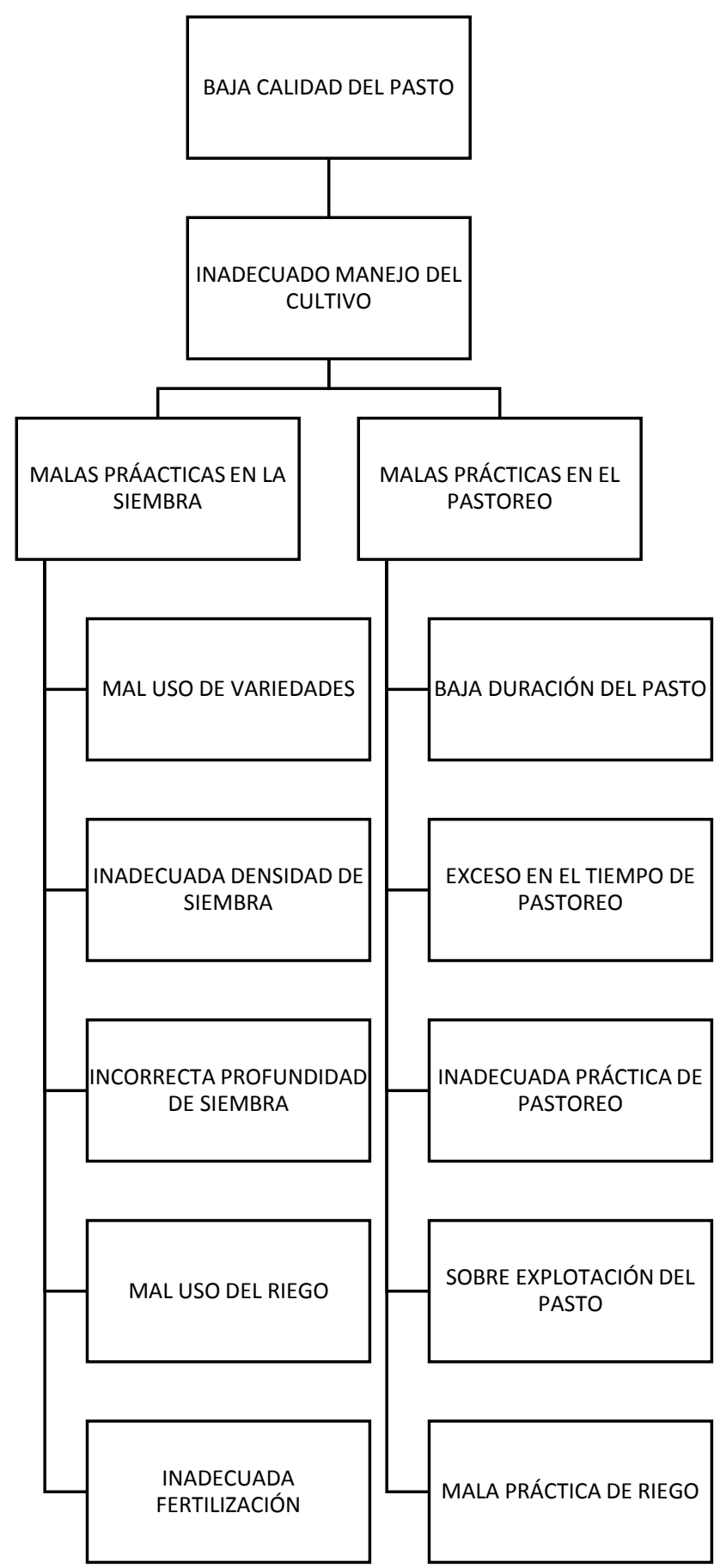

Figura 1. Análisis de problemas con relación al cultivo del rye grass en la microcuenca del río Chimborazo

Fuente: Talleres participativos y evaluación del nivel tecnológico

Elaborado por: Muñoz E., Moreno F. y Coello M., Cruz César

\section{Análisis de objetivos}

Igualmente con la participación de los involucrados con base en técnicas sencillas, se transformó la situación problémica anterior en una situación de futura deseada y alcanzable, determinándose los objetivos a lograr con la propuesta, el problema central se transformó en propósito, formulándose de 
la siguiente manera: adecuado manejo del cultivo, para lo cual se requieren 2 aspectos principales: adecuadas prácticas en la siembra y adecuadas prácticas en el manejo; para su cumplimiento el primero requiere de la adecuada profundidad de siembra, de buenas prácticas de riego y adecuada fertilización. Las adecuadas prácticas en el manejo se posibilitan por uso de pasto adecuado con relación al tiempo, control del sobrepastoreo y adecuadas prácticas de riego, como se puede observar en la figura 2.

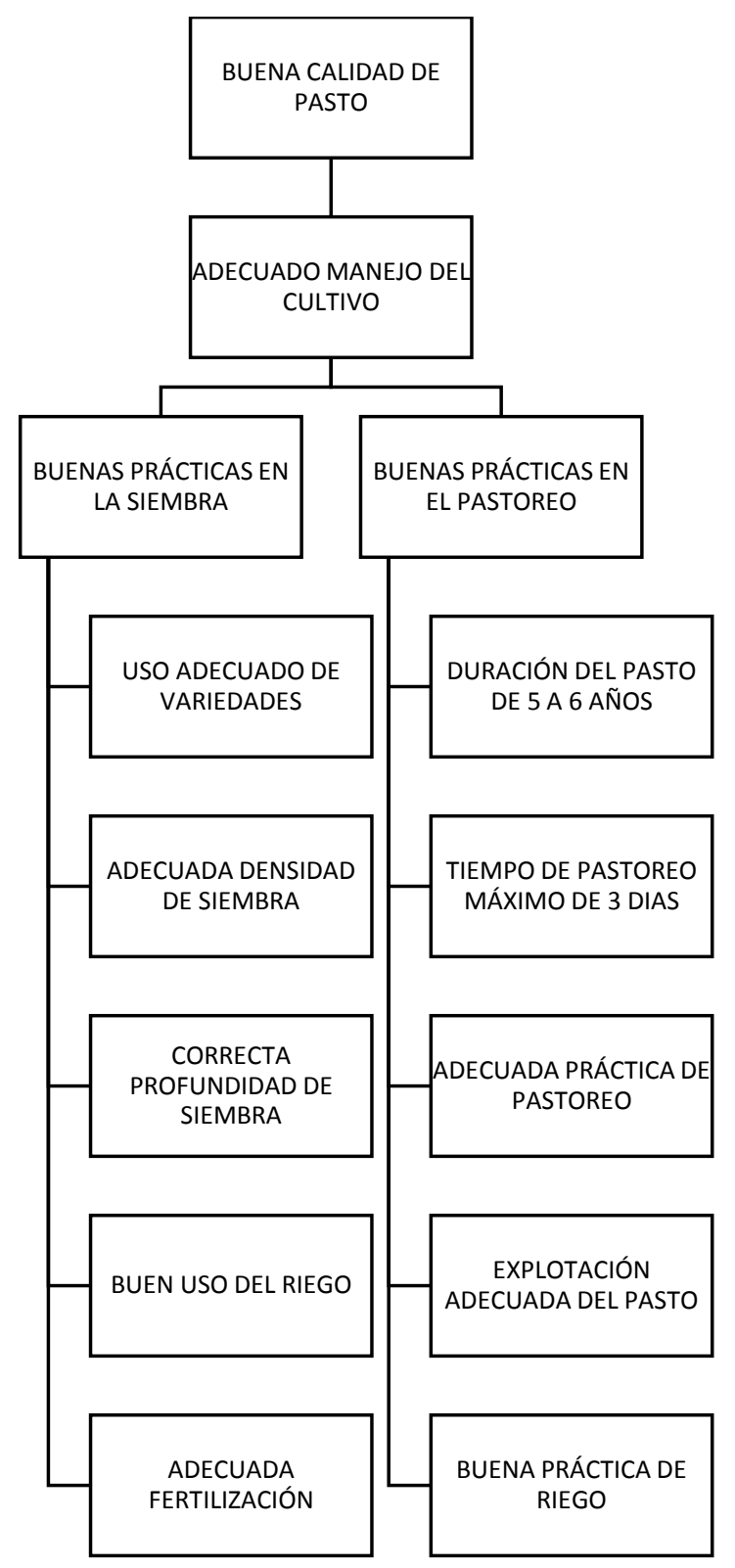

Figura 2. Análisis de objetivos para mejorar la producción del cultivo del rye grass en la microcuenca del río Chimborazo

Fuente: Talleres participativos y evaluación del nivel tecnológico

Elaborado por: Muñoz E., Moreno F. y Coello M., Cruz César 


\section{Análisis de alternativas}

Para el logro de los objetivos participativamente se definieron las siguientes alternativas:

a. Investigación y capacitación en prácticas agronómicas sostenibles

b. Asesoramiento técnico

\section{Análisis de involucrados}

Identificadas las alternativas de solución, mismas que pasan a constituirse en las estrategias de la propuesta, dentro del total de involucrados se identificaron a aquellos que en forma efectiva podrían contribuir con la ejecución de la misma. El análisis se realizó con base en lo que se indica en la tabla 18, en la cual inicialmente se caracterizan los involucrados a través de la función e intereses que persiguen. Posteriormente se hicieron los apuntes más importantes sobre el potencial, es decir, aquellos aspectos positivos que poseen y con los cuales pueden contribuir a poner en práctica la propuesta. Luego se analizaron las limitaciones, entendidas como aquellos aspectos que impiden la participación de los involucrados, para finalmente establecer las implicaciones, es decir, considerar los aspectos de la propuesta a nivel de proyecto que deben cumplirse para superar las limitaciones y aprovechar el potencial de los involucrados. Se debe señalar que las limitaciones no son una repetición de los problemas reportados en la evaluación del nivel tecnológico. 
Tabla 18. Análisis de involucrados

\begin{tabular}{|c|c|c|c|c|c|}
\hline INSTITUCIÓN & FUNCIÓN & INTERESES & POTENCIAL & LIMITACIÓN & IMPLICACIONE \\
\hline $\begin{array}{l}\text { Escuela } \\
\text { Superior Politécnica de Chimbora }\end{array}$ & $\begin{array}{l}\text { Investigación } \\
\text { Vinculación }\end{array}$ & $\begin{array}{l}\text { Desarrollar } \\
\text { conocimiento } \\
\text { Servir a la } \\
\text { colectividad }\end{array}$ & $\begin{array}{l}\text { Conocimiento } \\
\text { Personal }\end{array}$ & Movilización & $\begin{array}{l}\text { Asegurar la } \\
\text { movilización }\end{array}$ \\
\hline $\begin{array}{l}\text { Gobierno Autónomo } \\
\text { Descentralizado de la Parroquia } \\
\text { Juan }\end{array}$ & $\begin{array}{l}\text { Administración } \\
\text { de la } \\
\text { microcuenca }\end{array}$ & $\begin{array}{l}\text { Incentivar el desarrollo } \\
\text { de actividades } \\
\text { productivas comunitarias }\end{array}$ & $\begin{array}{l}\text { Recursos } \\
\text { Liderazgo }\end{array}$ & $\begin{array}{l}\text { Falta de } \\
\text { Técnicos }\end{array}$ & $\begin{array}{l}\text { Asegurar } \\
\text { los técnicos }\end{array}$ \\
\hline $\begin{array}{l}\text { Ministerio de Agricultura, } \\
\text { Acuacultura y Pesca }\end{array}$ & $\begin{array}{l}\text { Asesoría y } \\
\text { Apoyo }\end{array}$ & $\begin{array}{l}\text { Promover el } \\
\text { desarrollo } \\
\text { sostenible de la } \\
\text { producción y productivida } \\
\text { del } \\
\text { sector rural }\end{array}$ & Recursos Técn & Tecnología & $\begin{array}{l}\text { Capacitar } \\
\text { Técnicos }\end{array}$ \\
\hline $\begin{array}{l}\text { Instituto de Investigaciones } \\
\text { Agropecuarias }\end{array}$ & $\begin{array}{l}\text { Investigación y } \\
\text { Asesoría técnica } \\
\text { Científica }\end{array}$ & $\begin{array}{l}\text { Proporcionar } \\
\text { tecnología y } \\
\text { servicios } \\
\text { especializados } \\
\text { para impulsar la innovacić } \\
\text { agropecuaria } \\
\text { nacional }\end{array}$ & Personal & $\begin{array}{l}\text { Pocos } \\
\text { técnicos }\end{array}$ & $\begin{array}{l}\text { Asegurar } \\
\text { los técnicos }\end{array}$ \\
\hline Agricultores & $\begin{array}{l}\text { Cultivar la tierra } \\
\text { crianza de } \\
\text { animales }\end{array}$ & $\begin{array}{l}\text { Económicos } \\
\text { Sociales. }\end{array}$ & $\begin{array}{l}\text { Conocimiento } \\
\text { ancestrales }\end{array}$ & $\begin{array}{l}\text { Escasos } \\
\text { recursos } \\
\text { económicos } \\
\text { Escasos } \\
\text { conocimientos } \\
\text { desde el } \\
\text { enfoque de la } \\
\text { revolución } \\
\text { verde }\end{array}$ & $\begin{array}{l}\text { Investigar } \\
\text { prácticas } \\
\text { ancestrales } \\
\text { Generar } \\
\text { conocimientos } \\
\text { bajo enfoques } \\
\text { sostenibles, } \\
\text { sustentables y } \\
\text { accesibles para } \\
\text { agricultores } \\
\text { Asistir } \\
\text { técnicamente }\end{array}$ \\
\hline
\end{tabular}

Fuente: Talleres participativos

Elaborado por: Muñoz E., Moreno F. y Coello M., Cruz CésarMarco lógico

Con base en los análisis anteriores y en función del compromiso de los involucrados se elaboró la matriz de la propuesta, que sintetiza lo que se debe ejecutar para mejorar en la implementación y en el manejo del cultivo en estudio. 
Cuadro 19. Marco lógico para mejorar el manejo agronómico del cultivo del rye grass en la microcuenca del río Chimborazo.

Fuente: Talleres participativos

Elaborado por: Muñoz E., Moreno F. y Coello M. Cruz César

\begin{tabular}{|c|c|c|}
\hline NARRATIVA DE OBJETIVOS & & INDICADORES \\
\hline $\begin{array}{l}\text { Fin: Contribuir a la producción } \\
\text { de pasto de buena calidad }\end{array}$ & 1.1 & Se mejora en un $80 \%$ la producción de pasto en los próximos 5 años \\
\hline $\begin{array}{l}\text { Propósito: Mejora el manejo } \\
\text { del cultivo }\end{array}$ & 1.1 & El $80 \%$ de las familias de la microcuenca aplican prácticas adecuadas \\
\hline \multicolumn{3}{|l|}{ Componentes } \\
\hline \multirow[t]{4}{*}{ Investigación y capacitación } & $\begin{array}{l}1.1 \\
1.2\end{array}$ & $\begin{array}{l}\text { Se desarrolla un paquete tecnológico con prácticas sostenibles en } \\
\text { base a investigación participativa }\end{array}$ \\
\hline & 1.3 & $\begin{array}{l}\text { Se realiza un curso de capacitación por mes en manejo de pastos } \\
\text { para } 30 \text { jefes de familia, hasta cubrir al } 80 \% \text { de agricultores. }\end{array}$ \\
\hline & 1.4 & $\begin{array}{l}\text { Se implementan } 9 \text { ensayos demostrativos en campo en cada una } \\
\text { de las comunidades de la microcuenca. }\end{array}$ \\
\hline & 1.5 & Se efectúa una visita de campo por mes a los ensayos demostrativos. \\
\hline Asesoramiento técnico & 2.1 & $\begin{array}{l}\text { Se da asistencia técnica diaria y permanente con un técnico } \\
\text { por } 3 \text { comunidades }\end{array}$ \\
\hline
\end{tabular}

\section{Conclusiones}

La metodología desarrollada en forma participativa involucró principalmente a los agricultores; su aplicación es sencilla, puede detectar deficiencias en el proceso productivo del Rye Grass y posibilitar una planificación rápida para enfrentar la problemática identificada con precisión en cada práctica que afecta al cultivo de este pasto. Para ello se utiliza asistencia técnica y, de ser necesario, nuevas investigaciones.

Revisadas las prácticas agronómicas realizadas por los agricultores de la microcuenca del río Chimborazo para el cultivo del Rye Grass, la fase siembra acusa serias limitaciones, apenas $3 \%$ de los agricultores logra una calificación de excelente, en la fase de manejo o pastoreo $10 \%$ alcanza esta calificación, lo que afecta a la sostenibilidad del cultivo y limita la producción lechera, limitando los pastos bien manejados y de buena calidad.

El nivel tecnológico del cultivo en general del Rye Grass es calificado por la mayoría de los agricultores como regular y malo, lo que implica desarrollar investigaciones participativas, 
articulando decisores internos y externos a la microcuenca del río Chimborazo en la generación de tecnologías adecuadas a la realidad campesina de microcuencas andinas y que puedan ser adoptadas por los agricultores.

Se propone trabajar en asistencia técnica, capacitación e investigación con base en la problemática detectada y en función del marco lógico que recoge la planificación participativa realizada con los agricultores

\section{Bibliografía}

Alarcón, Z. (2007). Producción de forraje verde para ganado bovino en invierno. Reporte de resultados primer año. Instituto de Investigación y Capacitación Agropecuaria, Acuícola y Forestal del Estado de México. Universidad Autónoma de Chapingo, México, p. 58.

Clementeviven (2010). "Ray grass". Disponible en: http://blog.clementeviven.com.

Ducos, E. (2011). Densidad de siembra". Disponible en: http://usuarios.advance.com.ar/elcencerro/

Gobierno Autónomo Descentralizado de La Parroquia San Juan (2012). Plan de desarrollo y ordenamiento territorial de la Parroquia San Juan, 2012-2021.

Hidalgo, E. (2010). Proyecto de factibilidad para la importación y comercialización de semillas de pasto para la empresa H. AGROSEF S.A., que lleva a cabo sus actividades comerciales en las provincias de Carchi, Pichincha y Cotopaxi”. Quito-Ecuador. Repositorio de Tesis de Grado de la Universidad Tecnológica Equinoccial.

Masal (2009). "Manejo Sostenible de Suelos y Agua en Laderas". Disponible en: http://www.bvcooperacion.pe/biblioteca/bitstream/123456789/5100/1/BVCI0004468.pdf

ROCALBA (2011). "Semillas forrajeras y pratenses". Disponible en: http://www.rocalba.com.

Sian (2011). "La producción de pastos de altura. Kikuyo y Rye grass perenne en el estado Mérida”. Disponible en: http://sian.inia.gob.ve. 
Unión Ganadera Regional de Jalisco (2011). EL zacate Rye Grass Anual o Ballico Italiano”. México. Disponible en: http://www.ugrj.org.mx.

Universidad Pública de Navarra (2011). "Flora pratense y forrajera cultivada de la Península Ibérica”. Disponible en: http://www.unavarra.es.

Valencia, E. (2010). "Pastoreo". Disponible en: http://avalon.cuautitlan2.unam.mx/vaqu illas/manuales/manual_pastoreo.pdf 